experienced gynecologic pathologist. Data were retrieved from the medical records, including gynecologic data, sonographic findings, surgical and pathological results. The prevalence of endometrial carcinoma in such patients was calculated. Various characteristics were used to determine the associated risk factors. Results: Ninety-eight patients were analyzed. Twenty-nine patients (29.6\%) were subsequently diagnosed with stage I endometrial carcinoma after hysterectomy. The hysterectomy pathologies of 69 patients (70.4\%) were EIN and other benign diagnoses. The majority of patients who diagnosed with endometrial carcinoma were pre-menopause $(80 \%, \mathrm{p}=0.458)$ and nulliparous $(70 \%, \mathrm{p}=0.045)$. Endometrial thickness greater than $2 \mathrm{~cm}$ and body mass index (BMI) greater than $30 \mathrm{~kg} / \mathrm{m}^{2}$ were demonstrated as two associated risk factors for occult endometrial carcinoma with the odds ratio of 9.7 and 2.5, respectively.

Conclusion: Occult endometrial carcinoma was observed in approximately one-third of the patients with pre-operative EIN diagnosis. Endometrial thickness greater than $2 \mathrm{~cm}$ and BMI greater than $30 \mathrm{~kg} / \mathrm{m}^{2}$ were associated risk factors for occult endometrial carcinoma.

Poster (E17)

Endometrial Hyperplasia, Endometrial Intra-epithelial Neoplasia, and Endometrial Cancer

https://doi.org/10.3802/jgo.2021.32.S1.E17

\section{A window of opportunity study of nivolumab in early-stage endometrial cancer patients with MMRd who had failure after progestins for fertility-sparing treatment (NIVEC)}

Young Hwa Kwak, Junsik Park, Yun-Jung Go, Sunghoon Kim, Jung-Yun Lee*

Yonsei University College of Medicine, Seoul, Korea (JUNGYUNLEE@yuhs.ac)

Background: National Comprehensive Cancer Network guidelines recommend high-dose progesterone for fertilitysparing treatment only for patients with clinical stage IA, grade 1 without myometrial invasion. In patients with treatment failure from progestins, there is no treatment option except hysterectomy, even if the patients want to preserve the uterus. Recently, some studies evaluated the role of prognostic significance of the Proactive Molecular Risk Classifier for endometrial carcinoma classification infertility-sparing management of endometrial cancer. They showed that patients with mismatch repair deficiency (MMRd) had a lower response rate than those with mismatch repair proficiency with hormone therapy. MMRd tumors are known to be highly immunogenic and of great interest for immune checkpoint inhibitor. Results from a phase I/II study (CA209003/MDX1106-03) indicate that nivolumab (BMS-936558; anti-PD-1 monoclonal antibody) is active in multiple tumor types. In this regard, we suggest a single-arm phase II study investing the efficacy of nivolumab in the fertility-sparing management of endometrial cancer patients with MMRd who had failure with progestins.

Methods: Early-stage endometrial cancer patients with MMRd who had failure after progestins for fertility-sparing treatment and desire to preserve fertility was offered nivolumab. Approximately 8 patients will be included from 6 centers in Korea for 3 years. The primary endpoint of the study is to determine the efficacy of nivolumab by assessment of complete response.

Poster (E18)

Endometrial Hyperplasia, Endometrial Intra-epithelial Neoplasia, and Endometrial Cancer

https://doi.org/10.3802/jgo.2021.32.S1.E18

\section{Prognostic factor of pretreatment thrombocytosis to endometrial cancer in Bhumibol Adulyadej Hospital}

\section{Arun Jevae, ${ }^{1}$ Piyawan Pariyawateekul,, ${ }^{1, *}$ Worrawan Sirichai, ${ }^{1}$ Kornkarn Bhamarapravatana, ${ }^{2}$ Komsun Suwannarurk ${ }^{3}$}

'Bhumibol Adulyadej Hospital, Bangkok, Thailand (nootpiya@gmail.com) ${ }^{2}$ Thammasat University, Bangkok, Thailand

${ }^{3}$ Thammasat University Hospital, Khlong Nueng, Thailand

Background: Endometrial cancer (EC) is the 6th most frequent of cancer in the world. GLOBOCAN in year 2020 reported that 417,367 new cases of endometrial cancer worldwide. In Thailand, EC is the 6th most frequent cancer after breast, colon, cervix, liver, and lung cancers. There were 4,524 new cases of EC in Thai women in year 2020. Current treatment of EC is total hysterectomy with bilateral salpingo-oopherectomy. Bilateral pelvic lymphadenectomy, omentectomy, and paraaortic lymph node (LN) sampling were needed for surgical staging in EC. Adjuvant radiotherapy or concurrent chemoradiation (CCRT) was designed after corrected staging of EC. According to the International Federation of Gynecology and Obstetrics (FIGO) stage, histopathology, histological grading, depth of myometrial invasion (MI) and present of lymph-vascular invasion (LVSI) were the prognostic factors of EC. Previous studies had demonstrated that thrombocytosis was the poor prognostic factor of many cancer including ovary, cervix, vulvar, and breast. Thrombocytosis was also the prognostic factor in EC that affected disease-free survival (DFS) and overall survival (OS). In the present study, the association of pretreatment 
thrombocytosis and other prognostic factors DFS and OS was the aim of study.

Methods: Aim of this study was to analyze the impact of pretreatment thrombocytosis to the prognosis of EC cases. Medical records of EC cases who underwent complete surgical staging, treatment and follow up in Bhumibol Adulyadej Hospital (BAH) during January 2008 and December 2020 were reviewed. Demographic data included age, body weight, height, body mass index, histology and grading, cancer stage, MI, LN metastasis, LVSI, peritoneal washing cytology, platelet count, recurrence, death and follow up time were collected. Associations of thrombocytosis and prognostic factors were analyzed with DFS and OS. A total of 222 cases were included in study. Mean age of participant in the present study was 56.16 years old. One third (88/222) of cases had age more than 60 years old. Ninety percent of histopathology was reported as endometrioid subtype. MI more than half of thickness, well and moderate differentiation (G1, 2) and advanced cancer stage (FIGO stage III, IV) were reported at $44.1 \%, 79.4 \%$, and $22.5 \%$, respectively. Thrombocytosis was reported at $8.1 \%$. Aggressive histopathology, advanced cancer stage (FIGO stage III, IV), MI more than half of thickness, LN metastasis, present of LVSI and thrombocytosis was significantly correlated with DFS. Advanced cancer stage (FIGO stage III, IV) and thrombocytosis had significant adverse effect on OS. In conclusion, advanced endometrial cancer stage and thrombocytosis were significant adverse prognostic factor.

Poster (E19)

Endometrial Hyperplasia, Endometrial Intra-epithelial Neoplasia, and Endometrial Cancer

https://doi.org/10.3802/jgo.2021.32.S1.E19

\section{The utility of preoperative serum CA125 combined with HE4 to predict lymph node metastasis in endometrial cancer: a cross- sectional study}

\footnotetext{
Sansanee Angsathapon, Nisa Pruksaritanond

Rajavithi Hospital, Bangkok, Thailand (nisa3054@gmail.com)
}

Objective: To investigate the diagnostic performance of the serum cancer antigen 125 (CA125), human epididymis protein 4 (HE4), a combination of CA125 and HE4, and a risk of ovarian malignancy algorithm (ROMA) in the pre-operative prediction of high risk of lymph node metastasis in patients with earlystage endometrial cancer (EC).

Methods: A cross-sectional study of data for patients with early-stage endometrioid EC treated surgically at Rajavithi
Hospital between April 2020 and April 2021 was commenced. The preoperative serum levels of CA125 and HE4 were measured and analyzed by clinicopathological characteristics. The receiver operating characteristic (ROC) curves were generated to determine the optimal cutoff values of CA125, HE4, and ROMA levels with optimum sensitivity and specificity for predicting lymph node metastasis.

Results: Eighty-six patients with surgically staged EC were identified. Lymph node involvement was detected in 9 patients $(10.5 \%)$ with a systemic lymph node dissection. The median serum CA125, HE4, and ROMA levels were significantly higher in EC patients with the presence of lymph node metastasis than those who did not $(\mathrm{p}<0.05)$. Based on the ROC curve, both serum markers showed good discrimination for the prediction of lymph node metastasis, with an optimal cutoff value of $35 \mathrm{U} /$ $\mathrm{mL}$ for CA125 (area under curve [AUC] $=0.789 ; 95 \%$ confidence interval $[\mathrm{CI}]=0.647-0.932), 200 \mathrm{pMol} / \mathrm{L}$ for HE4 (AUC=0.825; $95 \% \mathrm{CI}=0.700-0.950$ ), and $60 \%$ for ROMA (AUC $=0.856 ; 95 \%$ $\mathrm{CI}=0.720-0.982)$. At the optimal cutoff value, HE4 showed the highest sensitivity $(88.9 \%)$ whereas the combination of CA125 and HE4 had the highest specificity $(87.0 \%)$.

Conclusion: Pre-operative combining CA125 and HE4 level is associated with an increased risk of having lymph node metastasis in patients with early-stage EC. This biomarker panel can be used as a predictive marker and guidance for identifying EC patients who might most benefit from full staging surgery with lymphadenectomy.

Poster (E20)

Endometrial Hyperplasia, Endometrial Intra-epithelial Neoplasia, and Endometrial Cancer https://doi.org/10.3802/jgo.2021.32.S1.E20

\section{A personalised predictive 3-year overall survival for patients with uterine carcinosarcoma at tertiary care hospital of southern Thailand}

\section{Ingporn Jiamset," Pacharadol Taweerat}

Prince of Songkla University, Songkhla, Thailand (ing_ing01@hotmail.com)

Objective: To develop a 3-year overall survival (OS) nomogram to predict outcome of surgically staged patients with uterine carcinosarcomas (UCSs).

Methods: This retrospective study, the clinicopathological, treatment data, and oncological outcomes of 69 UCSs patients between January 2002 and September 2018 were analyzed. We identified and integrated significant prognostic factors for OS to develop a nomogram. The concordance probability (CP) was 